\title{
L'Heritage merita un Progetto Strategico Nazionale
}

La scelta di utilizzare il termine Heritage nel titolo del Convegno di Termoli è dovuta alla capacità di questo vocabolo di raccogliere in sé sinteticamente tutto ciò che si intende per eredità culturale.

Si tratta di un patrimonio esteso a dimensione di paesaggio e comprensivo di "ogni testimonianza di civiltà" materiale e immateriale, artistica e culturale, inclusi i valori, le tradizioni, i saperi, le abilità, i simboli e i gusti trasmessi anche tacitamente di generazione in generazione.

Ogni territorio è unico e irripetibile, caratterizzato dall'identità e dalla bellezza che lo contraddistinguono in relazione al tempo che lo ha attraversato nel combinarsi dell'agire della natura e del comportamento umano.

I suoi asset primari rientrano in gran parte nella categoria dei beni comuni, di quei beni che sono di uso non esclusivo.

$\mathrm{Su}$ di essi esercitano poteri decisionali soggetti molteplici, insistono interessi di varia dimensione e natura e si conducono attività di studio e analisi da parte di molti e con diverse prospettive.

Di qui la molteplicità di visioni in merito al valore che il patrimonio culturale possiede per il sistema Paese, per l'economia, per le comunità locali e per l'umanità.

La gestione del patrimonio culturale si caratterizza così per una elevata complessità connessa all'ordinamento giuridico e amministrativo e all'intreccio tra pubblico e privato in presenza di una miriade di soggetti agenti con obiettivi multipli. Soggetti connessi tra loro da legami deboli o inesistenti, al punto da apparire come una costellazione solo se "visti da lontano", rivelando invece le loro distanze nelle relazioni e nell'agire se osservati da vicino.

Soggetti eterogenei, tra cui le imprese che rivestono molteplici ruoli, da quello di produttori e fruitori culturali, a quello di ispiratori di logiche manageriali utili nella gestione dell'Heritage, a quello, infine, di possibili sponsor e mecenati.

In questo contesto, il Convegno si proponeva di valutare, in un confronto quanto più possibile interdisciplinare, se e come l'approccio imprenditoriale e manageriale potesse agevolare il percorso verso la valorizzazione del patrimonio culturale in un'ottica di sostenibilità economica, sociale e ambientale.

Varie risposte sono state raccolte al riguardo nelle due giornate di lavoro e alcune di queste sono pubblicate in questo numero della rivista.

$\mathrm{Al}$ riguardo, costruendo una riflessione a posteriori sui lavori presentati e sul dibattito che si è intrecciato nei giorni del convegno, come pure ripensando ad alcuni momenti preparatori dello stesso, sono rimbalzate alla mente tre parole che sembrano proporsi come ponti tra i concetti concatenati nel titolo del convegno "Heritage, management e impresa". Si tratta dei seguenti termini: memoria, viaggio, valore. 
sinergie Vol. 34, N. 99, 2016

La memoria è parte centrale del concetto stesso di Heritage nella sua visione di eredità culturale.

La memoria esprime la capacità di conservare, ricordare e rievocare mentalmente le esperienze del passato. Questo sia dal punto di vista personale che collettivo e storico.

Dal punto di vista personale la memoria conserva ciò che è entrato a far parte della conoscenza dell'individuo attraverso lo studio e le esperienze vissute.

La memoria collettiva preserva il patrimonio di valori comuni che definitisi nel tempo scolpiscono l'identità culturale di una comunità.

La memoria storica per parte sua raccoglie gli insegnamenti che una comunità ha fatto propri sulla base dellesperienza storica vissuta.

La memoria ha il compito di archiviare non solo i fatti e le scelte positive del passato, bensì anche e soprattutto di preservare il ricordo degli errori compiuti per farne tesoro nella progettazione del futuro.

Ma alla memoria oggi si porta poca attenzione. Si è troppo presi da un presente ingombrante e amplificato da una tecnologia che vive sull'istante e la velocità accorciando sempre più l'arco temporale cui fare riferimento, al punto che anche ciascuna notizia, per quanto singolare e rilevante, è fagocitata dalla notizia successiva nel giro di poche ore. Ne risulta un presente dilatato in una miope visione di breve periodo nella quale ci si rifugia perché non cè tempo per ricordare e si ha paura di alzare lo sguardo verso un futuro che intimorisce per la complessa indeterminatezza che lo caratterizza.

Forse il fermarsi a riflettere sullesperienza delle imprese storiche, a loro volta parte dell'Heritage di un Paese, potrebbe condurre ad una rilegittimazione della memoria nei processi evolutivi delle imprese, pur nella consapevolezza che il futuro mai possa tradursi in una semplice riproposizione del passato. Ed in proposito, come più volte osservato, piace ricordare la bella immagine che Richard Normann associa all'imprenditore quando lo rappresenta nel suo agire "qui e ora" ma nel suo dover essere contemporaneamente esule del passato e visitatore del futuro.

L'imprenditore, invero, da questo punto di vista appare come un viaggiatore. Un viaggiatore nel tempo per la capacità che deve manifestare di saper agire nel presente per la costruzione di un futuro desiderato, non dimentico però delle esperienze passate. Ma l'imprenditore è anche un viaggiatore nel senso autentico del termine di chi si muove, di chi si sposta da un punto ad un altro, nella direzione del raggiungimento delle mete assunte instradato dal sogno che ha generato la sua iniziativa.

Il viaggio, in realtà, potrebbe metaforicamente ben rappresentare l'impresa quale soggetto in movimento che tende a spostarsi da un punto ad un altro per raggiungere gli obiettivi che le sono propri.

Viaggiare, infatti, significa spostarsi, significa esplorare, significa rischiare, significa aprirsi al nuovo e alla diversità, significa umilmente riconoscere i "limiti della propria comprensione, la precarietà degli schemi e degli strumenti con cui una persona o una cultura presumono di capire o giudicano un'altra" (Claudio Magris), significa mantenere sempre aperta la porta della curiosità e per questa via dell'innovazione. 
Il viaggio richiede una progettazione che si avvia sulla scia di un sogno da realizzare. Introduce un puntuale senso di sfida per tutti i rischi che porta con sé indipendentemente dalla durata e dall'entità dello spostamento, anche se questi si arricchiscono in varietà ed entità più si passa da percorsi brevi e conosciuti a percorsi lunghi e sconosciuti da esplorare.

Il viaggio stimola la creatività e l'improvvisazione chiamata in causa per la soluzione degli innumerevoli problemi che si presenteranno durante il viaggio con la necessità di porre mano alla progettazione originale che lascia il posto ad una formulazione emergente dalla nuove strade sulle quali il cammino si dipana.

Apre all'arricchimento in conoscenza dell'alterità per la possibilità che dà di calarsi, pur con tutte le difficoltà del caso, nella realtà e nella cultura del luogo che si sta visitando.

Ridà spazio allo stupore e alla meraviglia nell'osservazione di ciò che si incontra nel cammino, emozioni che si pongono come puntuali precondizioni dell'innovazione. Stupore e meraviglia che potrebbero ovviamente ben germogliare anche nei luoghi in cui si risiede, nel momento in cui si riuscisse a sfuggire alla routine della quotidianità che impedisce di guardare al mondo con occhi nuovi.

Il viaggio insomma è una efficace metafora dell'impresa e ci appare fondante nel suo governo perché l'impresa è vita e il viaggio è vita e ascolto, socializzazione, arricchimento culturale, valorizzazione della diversità, curiosità, immaginazione, creatività, capacità di gestire l'imprevedibile, apprezzamento del valore sociale e culturale delle comunità e dei territori nei quali si agisce.

E tale metafora pone così in risalto il valore che le comunità e i territori, l'Heritage, possiedono per l'impresa.

Invero, il patrimonio artistico, culturale e ambientale di un Paese, e dell'Italia in particolare, produce valore in forma sia diretta che indiretta. Lo produce direttamente attraverso l'allestimento dei servizi connessi all'industria culturale e al turismo. Lo produce indirettamente in forma immateriale attraverso la forza che possiede di spargere e diffondere bellezza, cioè armonia, grazia, eleganza, stile, melodia, fascino, semplicità, gradevolezza, piacevolezza. Nel diffondere cioè tratti ambientali capaci di suscitare e amplificare la creatività nelle persone e nei territori, ponendosi come invisibile sostegno alla nascita e sviluppo dell'impresa.

L'Heritage da questo punto di vista costituisce un vero e proprio fattore produttivo disponibile a costo zero. Fattore che l'impresa però ha il dovere di remunerare, almeno parzialmente, per non trovarne depauperato il valore e per rinsaldare i rapporti con le comunità locali, nel rispetto del carattere di bene comune che l'Heritage possiede. L'impresa ha il dovere di rifondere, perlomeno in parte, il "debito figurativo" di tipo culturale verso l'Heritage, in ragione di un principio di sostenibilità che chiede di assicurare anche alle generazioni future il godimento dell'eredità culturale. E già alcune imprese hanno mosso decisi passi in questa direzione.

Ed è nella prospettiva del valore dell'Heritage per il Paese, per la comunità che lo costituisce e per le imprese che lo popolano, che s'intravvede la possibilità di creare un innovativo progetto, unalleanza tra pubblico e privato volta alla preservazione e valorizzazione dell'unicità e del bello del
Claudio Baccarani Gaetano M. Golinelli L'Heritage merita un Progetto Strategico Nazionale 
sinergie Vol. 34, N. 99, 2016

nostro Paese e delle sue produzioni.

Un Progetto Strategico Nazionale di marketing territoriale per la storia, la cultura e il turismo del Paese, che costituisca la base per interventi coerenti, non ispirati solo a logiche "spot", ma scaturenti da una visione unificante e comune che sappia portare a sistema le iniziative volte a preservare e valorizzare un patrimonio culturale senza uguali.

In un Paese dove la dimensione dell'Heritage presente sta di fatto creando una forte dispersione di interventi e iniziative, grande si avverte il bisogno di strategia.

Claudio Baccarani

Gaetano M. Golinelli

sinergie

italian journal of management DOI $10.7433 / \mathrm{s} 99.2016 .02$ pp. $15-18$

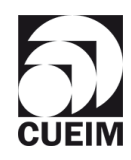

